\title{
Efficacy and Safety of PDM011011 Capsules as Compared to Metformin in Subjects with Type-2 Diabetes Mellitus: An Open-Label, Randomized, Active-Controlled, Multicentric, Phase III Study
}

\author{
Ashish C. Suthar1 ${ }^{*}$, Vikas G. Pai ${ }^{2}$, Yogesh Kadam³, Aniruddha Tongaonkar ${ }^{4}$, Shailaja Kale5, \\ Atul B. Deshpande6, Sharat Kolke7, Snehal Tanna8, Shrikant V. Deshpande9, \\ Purvi Chawla1, Debjit Biswas', Somesh Sharma1 \\ ${ }^{1}$ Piramal Enterprises Limited, Mumbai, India \\ ${ }^{2}$ Pai Clinic and Diagnostic Center, Pune, India \\ ${ }^{3}$ Poona Diabetes Centre, Pune, India \\ ${ }^{4}$ Tongaonkar Hospital, Pune, India \\ ${ }^{5}$ Orange Diabetes Clinical Research Unit, Pune, India \\ ${ }^{6}$ Ethika Clinical Research Centre Prakruti Hospital, Thane, India \\ ${ }^{7}$ Kolkes Clinic, Mumbai, India \\ ${ }^{8}$ Clinic De Care, Thane, India \\ ${ }^{9}$ Ashirwad Hospital and Medical Research Centre, Thane, India \\ Email: *ashish.suthar@piramal.com,paivikas@indiatimes.com,dryogeshkadam@gmail.com, \\ aniruddha_tongaonkar@yahoo.com,drshailaja@yahoo.com, atuldeshpande1104@yahoo.com, \\ sharatkolke@hotmail.com, clinicdecare@doctor.com, shrikantvdeshpande@rediffmail.com, \\ purvi.chawla@piramal.com, biswas_debjit@yahoo.com,somesh20032000@yahoo.com
}

Received 30 December 2015; accepted 29 January 2016; published 4 February 2016

Copyright (C) 2016 by authors and Scientific Research Publishing Inc.

This work is licensed under the Creative Commons Attribution International License (CC BY). http://creativecommons.org/licenses/by/4.0/

c) (i)

\section{Abstract}

Context: Bitter melon (Momoradica charantia) is one of the well-known plants used for lowering blood glucose since antiquity. Aims: To compare the efficacy and safety of PDM011011 capsule $(1200 \mathrm{mg} /$ day $)$ with Metformin $(1000 \mathrm{mg} /$ day) in a 15 weeks study using mean change in fasting plasma glucose (FPG) and Hb1Ac\% in subjects with type 2 diabetes mellitus (T2DM). Settings and

*Corresponding author.

How to cite this paper: Suthar, A.C., et al. (2016) Efficacy and Safety of PDM011011 Capsules as Compared to Metformin in Subjects with Type-2 Diabetes Mellitus: An Open-Label, Randomized, Active-Controlled, Multicentric, Phase III Study. Journal of Diabetes Mellitus, 6, 38-48. http://dx.doi.org/10.4236/jdm.2016.61005 
Design: This is an open-label, randomized, active-controlled, multicentric, phase III study. Methods and Material: A total of 123 eligible patients were randomized in 2:1 ratio in PDM011011 and Metformin arm. Total 83 subjects received PDM011011 capsule $(1200 \mathrm{mg} / \mathrm{day})$ and 40 subjects received Metformin $(1000 \mathrm{mg} /$ day) in their respective arms for 15 weeks. Subjects were analyzed for FPG and $\mathrm{Hb} 1 \mathrm{Ac} \%$ at baseline and during treatment visits (Visit 3 to Visit 7). Safety assessments were carried out. Results: In this study, the significant reduction in mean FPG level was observed after treatment with PDM011011 capsule and Metformin in T2DM patients. The mean change from baseline to week 15 in FPG was $14.52 \mathrm{mg} / \mathrm{dL}$ (95\% CI: 6.36, 22.67) in the PDM011011-treated subjects and $28.34 \mathrm{mg} / \mathrm{dL} \mathrm{(95 \%} \mathrm{CI:} \mathrm{21.35,} \mathrm{35.32)} \mathrm{in} \mathrm{the} \mathrm{Metformin-treated} \mathrm{subjects.} \mathrm{At} \mathrm{week} \mathrm{15,}$ the mean change from baseline in HbA1c levels was $0.27 \%(95 \%$ CI: $0.06,0.47)$ in the PDM011011 arm and $0.62 \%$ (95\% CI: 0.40, 0.83) in the Metformin arm. Conclusion: PDM011011 capsule (1200 $\mathrm{mg} /$ day $)$ exhibited the modest efficacy and safety as compared to Metformin $(1000 \mathrm{mg} /$ day $)$ in type 2 diabetes patients.

\section{Keywords}

Momoradica charantia, Glycebal, Diabetes mellitus, Anti-Diabetic

\section{Introduction}

Diabetes mellitus is a chronic metabolic disorder having multiple etiologies, characterized by hyperglycemia resulting from defective insulin secretion, resistance to insulin action, or a combination of both [1] [2]. In 2013, around 382 million people were diabetic and the number of diabetes cases is anticipated to be raised by $55 \%$ till 2035 [3]. Diabetes leads to 1.5 million deaths worldwide in 2012 and is the seventh leading global cause of death [4].

Diabetes is associated with many complications. Impaired renal function, blindness, amputations are the prominent microvascular complications of diabetes. Macrovascular complications affect the cardiovascular system manifesting myocardial infarction and strokes [5]. Pathophysiological conditions in Type 2 Diabetes mellitus (T2DM) are due to impaired insulin secretion by pancreatic $\beta$ cells and insulin resistance or both [6]. T2DM can be treated with metformin, insulin secretagogues, DPP-4 inhibitors, GLP-1 mimetic, acarbose and insulin [7]. For the management of T2DM, it is essential that treatment should improve glycemic control with no or least adverse effects [8].

Nowadays, medicinal plants are getting importance because of the better efficacy and less side effects than the artificial medication for the management of diabetes [9] [10]. Bitter melon (Momoradica charantia) is one of the well-known plants used for lowering blood glucose since antiquity [11]. Many preclinical studies and clinical studies have endorsed Momoradica charantia as a potential anti-diabetic agent [10] [12].

Recent studies show that bitter melon extract promotes a balanced storage of glucose in the liver as glycogen, supports healthy insulin secretion by the islets of Langerhans, and promotes peripheral glucose utilization and healthy serum protein levels according to in vivo and in vitro studies. Momordica charantia increases glucose utilization by liver, decrease gluconeogenesis via inhibition of two key enzymes glucose-6-phosphatase and fructose -1.6 bisphosphatase and improves glucose oxidation through the shunt pathway by activating glucose-6- phasphate dehydrogenase [13]. Momordica charantia restores hepatic glucose and lipid metabolism by increasing post-IR signal transduction linked to tyrosine phosphorylation of IRb and IRS (IR-insulin receptor) proteins thereby leading to PI3K activation [14].

Glycebal $^{\mathrm{TM}}$ (PDM011011) is a clinically validated blend prepared using the unique and patented "Juice Cap Technology" without use of heat, harmful chemicals and solvents. The blend is obtained from fresh unripe green bitter melon fruits grown in organic farms. PDM011011 capsules $\left(\right.$ Glycebal $^{\mathrm{TM}}$ ) is a new plant based oral medication for the management of diabetes and contains unique nucleoside complex (NEC) and Uridine as a chemical marker. Promising results were obtained in our previous clinical trial of PDM011011 capsules as compared to placebo in type 2 diabetes patients.

The objective of this study was to compare the efficacy and safety of PDM011011 capsule with Metformin in 
patients of type 2 diabetes mellitus.

\section{Materials and Methods}

\subsection{Patients}

\subsubsection{Inclusion Criteria}

Male and female subjects of age $\geq 30$ and $\leq 65$ years diagnosed with T2DM (based on American Diabetes Association criteria). Diagnosed subjects of T2DM not on any other anti-diabetic agent or taken any anti-diabetic agent for continuous period of 3 months in the past were screened. Subjects with fasting plasma glucose of $\geq 110$ $\mathrm{mg} / \mathrm{dL}$ and $\leq 250 \mathrm{mg} / \mathrm{dL}$; and $\mathrm{HbA} 1 \mathrm{c} \geq 7 \%$ and $\leq 10 \%$ at the time of randomization were included in the study. Females of child-bearing potential, willing to use adequate methods of contraception, were included in the study.

\subsubsection{Exclusion Criteria}

The subjects were excluded from study having Type 1 diabetes mellitus, secondary diabetes mellitus, pregnant or lactating women, clinically significant cardiac disease, endocrine abnormalities other than stable thyroid disease or patients requiring insulin therapy, serum creatinine of $\geq 1.5 \mathrm{mg} / \mathrm{dL}$, aspartate aminotransferase (AST) and alanine aminotransferase (ALT) values $\geq 2.5 \times$ upper limit of normal (ULN). In addition, subjects currently taking any other herbal products, dietary supplements, or medications during the past 8 weeks that could affect plasma glucose and/or body weight were excluded from the study.

The study was carried out in accordance with the ethical principles described in the Declaration of Helsinki. The Ethics Committee had reviewed the protocol and granted approval prior to the start of the study. Written informed consent form was taken from all subjects' prior to start of study procedure. This trial is registered at http://www.ctri.nic.in/ (CTRI number CTRI/2013/01/003308).

\subsection{Plant Material and Drug Preparation}

The unripe green fruits of Momordica charantia plant were used for preparation of dry juice powder i.e. Glycebal blend. It was authenticated at Piramal Enterprises Limited, Mumbai, India. The standardized dry juice powder was used to fill capsules. Each capsule contained $400 \mathrm{mg}$ of dry fruit juice powder with not less than 0.4 mg of Uridine as a chemical marker.

\subsection{Study Design}

This was an open-label, randomized, active-controlled, multicenter, phase III study to assess the efficacy and safety of PDM011011 capsules as compared to Metformin in subjects with T2DM. The study consisted of four phases viz. Screening phase (Visit 1, Day-21 to Day-8), Run-in Phase (Visit 2, Day-7 to Day-1), Treatment Phase (Visit 3 - Visit 7, Day 1 to Day 105), and Follow-up Phase (Visit 8, Day 112). In screening phase, 171 potential subjects were screened from 8 centers of India as per inclusion and exclusion criteria after obtaining written informed consent. The 123 eligible subjects were counselled for diet and exercise during the run-in period by a dietician/counsellor. Subjects were educated on the symptoms of hypoglycaemia and the method for self-monitoring of blood glucose level with the help of a glucose meter. Subjects were re-assessed at Visit 3 based on the FPG level. The eligible subjects were randomized to one of two treatment arm (Treatment A or Treatment B) in a 2:1 ratio. Subjects in the Treatment A arm $(\mathrm{N}=83)$ received one PDM011011 capsule of 400 mg twice daily half an hour before meals (one capsule each before breakfast and dinner). Subjects in the Treatment $\mathrm{B}$ arm $(\mathrm{N}=40)$ received one Metformin tablet $500 \mathrm{mg}$ twice daily during or after meals (one tablet each at breakfast and dinner).

Following randomization on Day 1, subjects returned for dose titration on Day 21. Subjects receiving PDM011011 capsules were advised to take an additional capsule of PDM011011 (400 mg) at night and subjects receiving Metformin tablets were advised to take an additional tablet of Metformin (500 mg) during or after lunch for the remaining treatment period. Subjects were called for outpatient visits on Days 49 (Visit 5), 77 (Visit 6), 105 (Visit 7) and 112 (Visit 8). During each Visit in the treatment phase (Visit 3 - Visit 7) safety and efficacy assessments were performed. Follow-up of all subjects in the study was taken at Visit 8 (Day 112). Out of 123,111 subjects completed the study successfully. 


\subsection{Efficacy and Safety Assessment}

Efficacy assessment was performed by mean change in FPG level (Visit 3 - Visit 7) and Hb1Ac \% (Visits 4 \& 7) from baseline. The primary efficacy outcome was mean change in the FPG level between PDM011011 capsules and Metformin from baseline to week 15 (Visit 7). The secondary efficacy outcome was mean change in the HbA1c between PDM011011 capsules and Metformin in these subjects. Safety assessment was performed by patient reported adverse events (Visit 2 - Visit 8), physical examination, vital signs, and concomitant medication (Visit 1 - Visit 8), safety laboratory measurements (Visits 2, 4, 6 \& 8), and ECG (Visits 2 \& 8).

\subsection{Randomization}

This was an open label, active controlled, randomized study. All eligible subjects were randomized in 2:1 ratio for the PDM011011 capsule or Metformin treatment arms respectively.

\subsection{Statistical Analysis}

Statistical analysis was carried out using statistical analysis system (SAS) 9.1.3 or higher.

Continuous variables (age, height, weight and BMI) were described by descriptive statistics (mean, SD) and categorical variables were presented by counts and percentages (race and gender). The mean change in FPG or HbA1c from baseline to Week 15 between PDM011011 capsules group and Metformin group were compared by an analysis of covariance (ANCOVA) model with treatment as fixed effect and baseline value of FPG or HbA1c as a covariate respectively. The mean change from baseline in FPG or HbA1c within PDM011011 capsules and Metformin group at each visit during treatment phase was analyzed using pair-t test. The difference between PDM011011 group and Metformin group 95\% CI was analyzed using Two sample t-test for mean change from baseline in FPG or HbA1c at each visit during treatment phase. For comparison of PDM011011 capsule and Metformin, the difference in least square means (LSM), corresponding standard error, two-sided 95\% Confidence Interval (CI), two sided p-value was presented. The non-inferiority of PDM011011 capsules was established if the upper limit of 2-sided 95\% CI was lower than the non-inferiority margin of - 25 by Satterthwaite estimate. In ANCOVA model the per-protocol population was used for FPG analysis while the intent-to-treat (ITT) and per-protocol (PP) population were used for the analysis of HbA1c.

\section{Results}

\subsection{Subject Disposition}

In this study, a total of 171 subjects were screened, out of which 123 subjects were enrolled from 8 centers of India. Since, 46 subjects failed in screening tests and 2 subjects were discontinued prior to randomization. The eligible subjects were randomized in 2:1 ratio and received either PDM011011 capsule $(\mathrm{N}=83)$ or Metformin tablet $(\mathrm{N}=40)$. A total of 111 subjects (90\% approximately) completed the study (Figure 1 , Table 1$)$. The most commonly reported reasons for study discontinuation were withdrawal of consent (5 subjects from PDM011011 arm) and subjects lost to follow-up (4 subjects; 1 subject from PDM011011 arm and 3 subjects from Metformin arm). Two subjects in PDM011011 arm discontinued for safety reasons as judged by the investigator and/or sponsor, due to the withdrawal criteria being met (blood glucose values $>270 \mathrm{mg} / \mathrm{dL}$ ).

\subsection{Demography}

The demographic characteristics are summarized in Table 2. The mean (SD) age of the subjects in the PDM011011 and Metformin arms was 49.43 years (9.26 years) and 47.70 years (9.11 years), respectively. All enrolled subjects were Asian. The proportions of male subjects were higher than female i.e. $55.4 \%$ and $57.5 \%$ males in the PDM011011 and Metformin arms, respectively. Both groups had almost similar mean height, mean weight, and mean body mass index (BMI).

\subsection{Medical and Surgical History}

At least one pre-existing medical condition was reported in 73.5\% (61/83) subjects in the PDM011011 arm and $77.5 \%$ (31/40) in the Metformin arm respectively. T2DM was reported as a pre-existing condition in 53.01\% 


\section{A. C. Suthar et al.}

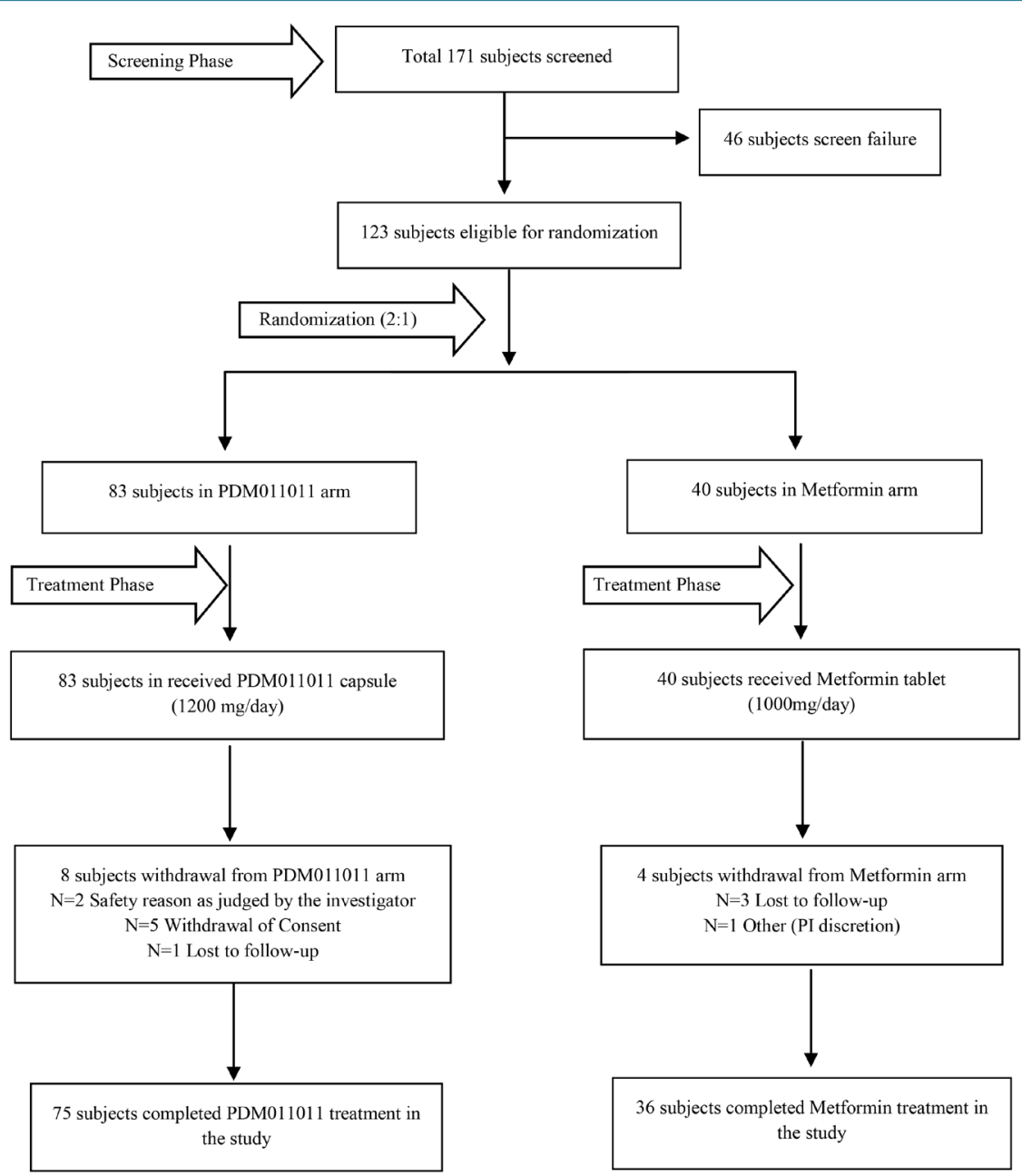

Figure 1. Consort diagram.

Table 1. Subject disposition with respect to the ITT and per protocol.

\begin{tabular}{|c|c|c|}
\hline Parameter & PDM011011 capsules $(\mathrm{N}=83)$ & Metformin $(\mathrm{N}=40)$ \\
\hline Subjects dosed & $83(100.00 \%)$ & $40(100.00 \%)$ \\
\hline Intent to treat population & $80(96.39 \%)$ & $39(97.50 \%)$ \\
\hline Per protocol population & $73(87.95 \%)$ & $35(87.50 \%)$ \\
\hline Subjects excluded from PP population with compliance $<80 \%$ & $2(2.40 \%)$ & $1(2.50 \%)$ \\
\hline \multicolumn{3}{|l|}{ Study completed } \\
\hline Yes & 75 (90.36\%) & $36(90.00 \%)$ \\
\hline No & $8(9.64 \%)$ & $4(10.00 \%)$ \\
\hline \multicolumn{3}{|l|}{ Reason for study termination } \\
\hline Prematurely discontinued & $8(9.64 \%)$ & $4(10.00 \%)$ \\
\hline Safety reason as judged by the investigator and/or Sponsor & $2(25.00 \%)$ & 0 \\
\hline Withdrawal of consent & $5(62.50 \%)$ & 0 \\
\hline Lost to follow-up & $1(12.50 \%)$ & $3(7.50 \%)$ \\
\hline Other, specify ${ }^{*}$ & 0 & $1(2.50 \%)$ \\
\hline on PI discretion $^{* *}$ & 0 & $1(2.50 \%)$ \\
\hline
\end{tabular}

*The denominator for percentage calculation is the number of subjects who prematurely discontinued. **The denominator for percentage calculation is the number of subjects who has other reason to be prematurely discontinued. 
Table 2. Demographics and disease characteristics (safety population).

\begin{tabular}{|c|c|c|}
\hline Parameter & PDM011011 capsules $(\mathrm{N}=83)$ & Metformin $(\mathrm{N}=40)$ \\
\hline \multicolumn{3}{|l|}{ Age (Years) } \\
\hline $\mathrm{N}$ & 83 & 40 \\
\hline Mean (SD) & $49.43(9.26)$ & $47.70(9.11)$ \\
\hline \multicolumn{3}{|l|}{ Race } \\
\hline Asian & 83 (100.00\%) & $40(100.00 \%)$ \\
\hline Black & 0 & 0 \\
\hline White/Caucasian & 0 & 0 \\
\hline Others & 0 & 0 \\
\hline \multicolumn{3}{|l|}{ Gender, n (\%) } \\
\hline Male & $46(55.42 \%)$ & $23(57.50 \%)$ \\
\hline Female & 37 (44.58\%) & $17(42.50 \%)$ \\
\hline \multicolumn{3}{|l|}{ Height $(\mathrm{cm})$} \\
\hline $\mathrm{N}$ & 83 & 40 \\
\hline Mean (SD) & $160.95(8.74)$ & $160.42(8.44)$ \\
\hline \multicolumn{3}{|l|}{ Weight (kg) } \\
\hline $\mathrm{N}$ & 83 & 40 \\
\hline Mean (SD) & $69.71(11.95)$ & 71.05 (9.99) \\
\hline \multicolumn{3}{|l|}{$\operatorname{BMI}\left(\mathrm{kg} / \mathrm{m}^{2}\right)$} \\
\hline $\mathrm{N}$ & 83 & 40 \\
\hline Mean (SD) & $26.96(4.46)$ & $27.62(3.49)$ \\
\hline \multicolumn{3}{|l|}{ Pre-existing medical condition } \\
\hline Type 2 diabetes mellitus & $44(53.01 \%)$ & $26(65.00 \%)$ \\
\hline Newly diagnosed Type 2 DM & 29 (46.99\%) & $14(35.00 \%)$ \\
\hline \multicolumn{3}{|l|}{ Co-morbidity } \\
\hline Hypertension & $20(24.10 \%)$ & $12(30.00 \%)$ \\
\hline Dyslipidemia & $7(8.43 \%)$ & $1(2.50 \%)$ \\
\hline Mean (SD) Fasting glucose level (mg/dl) & $155.35(35.00)$ & $147.63(29.82)$ \\
\hline Mean (SD) HbA1c (\%) & $8.29(1.02)$ & $8.08(1.01)$ \\
\hline
\end{tabular}

(44/83) of subjects in the PDM011011 arm and 65.0\% (26/40) of subjects in the Metformin arm. The remaining subjects were newly diagnosed T2DM patients. Hypertension was the most frequently reported co-morbidity followed by dyslipidemia in $24.1 \%$ and $8.43 \%$ subjects, respectively in the PDM011011 arm, and 30\% and $2.50 \%$ subjects, respectively in the Metformin arm. A total of 46 subjects (out of 83 subjects) in the PDM011011 arm and 20 subjects (out of 40 subjects) in the Metformin arm received at least one prior or concomitant medication. The most commonly reported concomitant medications were from the Anatomical Therapeutic Chemical (ATC) class of blood glucose lowering drugs.

\subsection{Efficacy Evaluation}

3.4.1. Effect on Fasting Plasma Glucose Level

Primary analysis was performed on Per Protocol (PP) population. At baseline, the mean (SD) FPG levels were 153.55 (35.00) mg/dL and 147.63 (29.82) mg/dL in the PDM011011 and Metformin arms, respectively. At week 
15 (visit 7), the mean (SD) FPG levels were 139.03 (32.56) mg/dL in the subjects of PDM011011 (1200 mg/day) treatment arm and 119.29 (20.15) $\mathrm{mg} / \mathrm{dL}$ in the subjects of Metformin (1000 mg/day) arm, respectively. The mean change from baseline to week 15 in FPG was $14.52 \mathrm{mg} / \mathrm{dL}$ (95\% CI: 6.36, 22.67) in the PDM011011treated subjects and $28.34 \mathrm{mg} / \mathrm{dL}$ (95\% CI: 21.35, 35.32) in the Metformin-treated subjects. The difference in mean change in FPG levels from baseline to week 15 between PDM011011 and Metformin was $-13.82 \mathrm{mg} / \mathrm{dL}$ (95\% CI: -24.42, -3.22) (Table 3).

The adjusted mean change in FPG levels from baseline to week 15 was $13.467 \mathrm{mg} / \mathrm{dL}$ in the PDM001001 and $30.545 \mathrm{mg} / \mathrm{dL}$ in the Metformin arms. The estimated mean difference in FPG levels between the treatment arms, PDM001001 and Metformin, from baseline to week 15 was $-17.078 \mathrm{mg} / \mathrm{dL}$ (95\% CI: $-27.347,-6.808)$. The pre-specified clinically acceptable difference for non-inferiority of PDM011011 to Metformin was -25 (Upper limit of the 95\% CI) (Table 4). Based on the analysis, although there was a statistically significant reduction in mean FPG levels in both the PDM011011 and Metformin arms from baseline to week 15, it may not be inferred that PDM011011 is non-inferior to Metformin in lowering the fasting plasma glucose in T2DM patients.

\subsubsection{Effect on HbA1c Levels}

In the PP population, at baseline, the mean (SD) HbA1c levels were 8.29\% (1.02) and 8.08\% (1.01), respectively in the PDM011011 and Metformin arms (Table 5). There was a reduction in the mean HbA1c level from baseline to week 3 (Visit 4) through week 15 (Visit 7), in both the treatment arms. At week 15, the mean change from baseline in HbA1c levels was $0.27 \%$ (95\% CI: 0.06, 0.47) in the PDM011011 arm and $0.62 \%$ (95\% CI: $0.40,0.83$ ) in the Metformin arm. The difference in mean change in HbA1c levels from baseline to week 15 between the PDM011011 and Metformin arms was $-0.34 \%$ (95\% CI: $-0.64,0.04)$. Similar reductions in HbA1c levels were observed in the ITT population.

\subsection{Safety Evaluation}

A total of 31 treatment emergent adverse events (TEAEs) were reported in 21 subjects, of which 22 treatment emergent AEs were reported in 14 (16.87\%) subjects in the PDM011011 arm whereas 9 TEAEs were reported in $7(17.50 \%)$ subjects in the Metformin arm. There was no clinically significant difference observed in the adverse events (AEs). Only two cases of serious adverse events (SAEs) was reported, one in each arm. Hypotension was reported in one subject in the PDM011011 arm and pyrexia was reported in one subject in the Metformin arm. However, these SAEs were considered as not related to the study drug. None of the AEs or SAEs led

Table 3. Summary of descriptive statistics for FPG (PP population).

\begin{tabular}{ccccc}
\hline $\begin{array}{c}\text { Parameter/Fasting } \\
\text { Plasma glucose (mg/d) }\end{array}$ & Statistics & $\begin{array}{c}\text { PDM011011 } \\
\mathbf{( N = 7 3 )}\end{array}$ & $\begin{array}{c}\text { Metformin } \\
\mathbf{( N = 3 5 )}\end{array}$ & $\begin{array}{c}\text { Difference between PDM011011 and } \\
\text { Metformin 95\% CI (Lower Cl, Upper CI) }\end{array}$ \\
\hline $\begin{array}{c}\text { Screening/Visit 1 } \\
\text { Visit 3a/Baseline }\end{array}$ & Mean (SD) & $153.69(35.57)$ & $144.46(25.97)$ & - \\
Visit 7 & Mean (SD) & $153.55(35.00)$ & $147.63(29.82)$ & - \\
$\begin{array}{c}\text { Change from } \\
\text { baseline to Visit 7 }\end{array}$ & $\begin{array}{c}\text { Mean (SD) } \\
\text { 95\% CI }\end{array}$ & $139.03(32.56)$ & $119.29(20.15)$ & $-13.82(-24.42)$ \\
\hline
\end{tabular}

*As a p-value for test of equality of variance is $<0.05$, Cl was calculated by Satterthwaite method.

Table 4. Summary of ANCOVA for FPG (PP population).

\begin{tabular}{|c|c|c|c|c|c|c|c|c|}
\hline \multirow{2}{*}{ Source } & \multirow{2}{*}{ DF } & \multirow{2}{*}{ F-value } & \multirow{2}{*}{$\begin{array}{l}\text { 2-sided } \\
\text { p-value }\end{array}$} & \multicolumn{2}{|c|}{ Least Square Means } & \multicolumn{3}{|c|}{$\begin{array}{l}\text { Estimate and } 2 \text {-sided } 95 \% \text { Cl for difference } \\
\text { PDM011011 capsules verses Metformin }\end{array}$} \\
\hline & & & & PDM011011 capsules & Metformin & Estimate & Lower bound & $\begin{array}{l}\text { Upper } \\
\text { bound }\end{array}$ \\
\hline Per protocol Set & & & & 13.467 & 30.545 & -17.078 & -27.347 & -6.808 \\
\hline Baseline FPG & 1 & 56.90 & $<0.0001$ & & & & & \\
\hline Treatment & 1 & 10.87 & 0.0013 & & & & & \\
\hline
\end{tabular}


Table 5. Summary of descriptive statistics for HbA1c.

\begin{tabular}{|c|c|c|c|c|}
\hline Parameter/ HbA1c (\%) & Statistics & PDM011011 & Metformin & $\begin{array}{c}\text { Difference between } \\
\text { PDM011011 and Metformin } \\
\text { 95\%CI (Lower CI, Upper CI) }\end{array}$ \\
\hline PP Population & $\mathbf{N}$ & 73 & 35 & - \\
\hline Baseline/Visit 1 & Mean (SD) & $8.29(1.02)$ & $8.08(1.01)$ & - \\
\hline Visit 4 (week 3) & Mean (SD) & $8.11(1.08)$ & $7.88(1.22)$ & \\
\hline Change from baseline to Visit 4 & $\begin{array}{c}\text { 95\% CI } \\
\text { (Lower Cl, Upper CI) }\end{array}$ & $0.05,0.30$ & $0.004,0.39$ & $-0.24,0.21^{*}$ \\
\hline Visit 7 (week 15) & Mean (SD) & $8.01(1.25)$ & $7.46(1.13)$ & - \\
\hline \multirow{2}{*}{ Change from baseline to Visit 7} & Mean (SD) & $0.27(0.89)$ & $0.62(0.63)$ & $-0.34(0.81)$ \\
\hline & $\begin{array}{c}\text { 95\% CI } \\
\text { (Lower Cl, Upper CI) }\end{array}$ & $0.06,0.47$ & $0.40,0.83$ & $-0.64,-0.04^{* *}$ \\
\hline \multicolumn{5}{|l|}{ ITT Population } \\
\hline \multirow[t]{2}{*}{ Baseline/Visit 1} & $\mathrm{~N}$ & 80 & 39 & - \\
\hline & Mean (SD) & $8.32(1.01)$ & $8.14(1.05)$ & - \\
\hline \multirow[t]{2}{*}{ Visit 4 (week 3) } & $\mathrm{N}$ & 80 & 39 & - \\
\hline & Mean (SD) & $8.12(1.05)$ & $7.94(1.25)$ & - \\
\hline \multirow[t]{3}{*}{ Change from baseline to Visit 4} & $\mathrm{~N}$ & 80 & 39 & - \\
\hline & Mean (SD) & $0.20(0.55)$ & $0.20(0.54)$ & $-0.003(0.55)$ \\
\hline & $\begin{array}{c}\text { 95\% CI } \\
\text { (Lower Cl, Upper CI) }\end{array}$ & $0.07,0.32$ & $0.02,0.37$ & $-0.21,0.21^{*}$ \\
\hline \multirow[t]{2}{*}{ Visit 7 (week 15) } & $\mathrm{N}$ & 75 & 36 & - \\
\hline & Mean (SD) & $8.03(1.24)$ & $7.51(1.15)$ & - \\
\hline \multirow[t]{3}{*}{ Change from baseline to Visit 7} & $\mathrm{~N}$ & 75 & 36 & - \\
\hline & Mean (SD) & $0.27(0.88)$ & $0.62(0.62)$ & $-0.34(0.80)$ \\
\hline & $\begin{array}{c}\text { 95\% CI } \\
\text { (Lower Cl, Upper CI) }\end{array}$ & $0.06,0.47$ & $0.41,0.82$ & $-0.63,-0.05^{* *}$ \\
\hline
\end{tabular}

*As a p-value for test of equality of variance is $<0.05, \mathrm{Cl}$ was calculated by Pooled method. ${ }^{* *}$ As a p-value for test of equality of variance is $>0.05$, $\mathrm{Cl}$ was calculated by Satterthwaite method.

to discontinuation of the subjects from the study. PDM011011 capsule and Metformin tablet were well tolerated during the study.

Majority of the TEAEs reported during the study, were judged as not related to the study treatments, PDM011011 and Metformin (overall -71\%, 22/31 TEAEs in 17 subjects), by the Investigator. All the TEAEs reported during the study were of mild or moderate intensity. No severe TEAEs were reported in any of the treatment arms.

There were no clinically significant changes observed in vital signs assessment, ECG evaluation or the laboratory test conducted in either treatment arms of this study.

\section{Discussion}

Type 2 diabetes mellitus is the most common metabolic disorder allied with microvascular and macrovascular complications [15]. Existing treatments are not always adequate in sustaining euglycemia and evading late stage diabetic complications [16]. One of the treatment options available for management of T2DM is insulin therapy but it is associated with several issues such as low patient compliance, hypoglycemia and weight gain [17]. Despite benefits and ease of administration, most of the oral anti-diabetic agents cause adverse effects including hypoglycemia, nausea, gastro-intestinal disturbances and possibly pancreatitis [18].

Metformin (biguanide derivative) is a potent oral hyperglycemic agent, now recommended as standard or 
first-choice agent for treatment of new-onset of type-2 diabetes [19]. The most common side effects of Metformin are gastrointestinal discomfort and diarrhea, while lactic acidosis and hypoglycemia are seen rarely [20].

Plant based medicines have an extensive prehistoric evidence of practice in various pathological disorders. Bitter melon (Momordica charantia) is traditionally used for the treatment of various diseases including diabetes [21]. A large number of preclinical and clinical studies have thus shown that Momordica charantia is able to lower plasma glucose levels. Various forms of bitter melon, including dried powder, fresh juice, bitter melon extract, and fried bitter melon have been used in different clinical trials [9].

In our previous study (Phase II), we found that statistical significant reduction in fasting glucose level (14.59\%) and postprandial blood sugar level (22.21\%) in T2DM patients, after treatment with PDM011011 capsule (1.2 g/day) for 3 months as compared to placebo capsule. The higher reduction in HbA1c\% was observed from baseline to end point in the patients receiving PDM011011 capsule than placebo treated group. Unique nucleoside complex (NEC) and Uridine contained in PDM011011 capsule may be responsible for reduction in HbA1c level.

In the present study, we assessed the efficacy and safety of the standardized form of bitter melon in treating T2DM patients compared to the standard of care i.e. Metformin. Fasting plasma glucose is a validated biomarker indicative of insulin resistance. The results indicated that significant reduction in the FPG level from baseline among the patients with type 2 diabetes who received PDM011011 capsule (1200 mg/day) and Metformin (1000 $\mathrm{mg} /$ day). Upon final analysis, it was observed that although there was a statistically significant reduction in mean FPG levels in both treatment arms from baseline to week 15, PDM011011 is not comparable to Metformin in lowering the FPG in type 2 diabetic patients. Faungchan et al. reported that significant reduction in fructosamine level from baseline in T2DM patients who received Metformin (1 g/day) and bitter melon fruit pulp powder (2000 mg/day) for 4 weeks. However, the effect was less as compared to $1 \mathrm{~g} /$ day dose of Metformin [11]. In another study, Rahman et al. mentioned that treatment of bitter melon powder ( $2 \mathrm{~g} /$ day and $4 \mathrm{~g} / \mathrm{day})$ for 10 weeks significantly reduced fasting blood glucose level in T2DM patients [22]. These results are similar with effect demonstrated by PDM011011 capsule even at a low dose of drug (1200 mg/day).

HbA1c is an important biomarker which represents mean plasma glucose in the past 8 - 12 weeks [23]. The baseline HbA1c values were similar in both treatment arms. Improvement in glycemic control, as evident from the reduction in mean HbA1c values at week 3 through week 15 when compared to baseline values, was observed in PDM011011 and Metformin group. At week 15, the reduction in mean HbA1c values compared to baseline values was $0.27 \%$ in the PDM011011 arm and 0.62\% in the Metformin arm. Dans et al. reported that treatment of bitter melon extract for 3 months in T2DM patients exhibited non-significant reduction in HbA1c\% levels [24]. In another study, Zankar et al. mentioned that statistically significant reduction was observed in $\mathrm{HbA} 1 \mathrm{c} \%$ from baseline in type 2 diabetes patients treated with bitter melon powder $(1 \mathrm{gm} /$ day $)$ for 4 months [25].

PDM011011 capsule (1200 mg/day) and Metformin (1000 mg/day) was well tolerated during the study. No other clinically significant changes were observed in the vital sign assessment, ECG evaluation and laboratory tests performed in the study period. The significant reduction in fasting glucose level and HbA1c\% confirms the anti-diabetic potential of PDM011011 capsule. However, the reduction was less as compared to Metformin.

Piramal Enterprises Limited (PEL) has developed a novel extraction process to obtain a standardized powder of dried juice enriched with bitters and proteins in the form of capsules. PDM011011 capsule (Glycebal ${ }^{\mathrm{TM}}$ ) is a new plant based oral medication for the management of diabetes. It contains unique nucleoside complex and Uridine as a chemical marker. Sugar nucleotides (especially UDP-glucose) play a role in the biosynthesis of glycogen and many other carbohydrate derivatives [26] [27]. UDP-glucose acts as the substrate for glycogen synthesis [28] [29]. The nucleotide sugar (uridine diphospho-N-acetylglucosamine) is also an essential intermediate in the synthesis of a wide array of complex glycans from sugar molecules which takes place by hexosamine biosynthesis pathway (HBP) [30].

Thus different nucleosides work in the liver to coordinate various enzymes that control blood glucose and other nutrients. We postulate that this type of 'nucleosides complex' including Uridine, present in our Momordica charantia capsule may potentiate the conversion of glucose or sugar molecules to glycogen and other glycan forms in liver and thus helps to regulate the blood glucose level.

\section{Conclusion}

PDM011011 capsule has been found to be reasonably safe and well-tolerated in this trial of 12 weeks treatment 
duration in 123 treatment naïve type 2 diabetes patients or who had not received anti-diabetic agent for at least a continuous period of 3 months prior to screening period. PDM011011 capsule did not appear to cause hypoglycemia as no event was reported in this clinical trial even with frequent self-monitoring of blood glucose. In term of efficacy, PDM011011 capsule appears to have the modest blood glucose lowering effect. Though PDM011011 exhibited less activity than Metformin, it has proven good blood sugar lowering activity in type 2 diabetes patients.

\section{Acknowledgements}

We are indebted to Dr. G. Tyebkhan for her expert comments and review of the manuscript, K. Salkar for administrative support and Karmic Life sciences LLP for drafting of the manuscript.

\section{Source(s) of Support}

No support taken from any organization/institution.

\section{Conflicting Interest}

We have no conflict of interest.

\section{References}

[1] American Diabetes Association (2008) Diagnosis and Classification of Diabetes Mellitus. Diabetes Care, 31, S55-S60. http://dx.doi.org/10.2337/dc08-s055

[2] Balasubramaniam, D., Mitra, A. and Mahadevappa, M. (2010) Antidiabetic and Hypolipidaemic Effects of Few Common Plants Extract in Type 2 Diabetic Patients at Bengal. International Journal of Diabetes and Metabolism, 18, 59-65.

[3] Guariguata, L., Whiting, D.R., Hambleton, I., Beagley, J., Linnenkamp, U. and Shaw, J.E. (2014) Global Estimates of Diabetes Prevalence for 2013 and Projections for 2035. Diabetes Research and Clinical Practice, 103, 137-149. http://dx.doi.org/10.1016/j.diabres.2013.11.002

[4] World Health Organization (2014) The Top 10 Causes of Death. WHO, Geneva. http://www.who.int/mediacentre/factsheets/fs310/en/.

[5] Forbes, J.M. and Cooper, M.E. (2013) Mechanisms of Diabetic Complications. Physiological Reviews, 93, $137-188$. http://dx.doi.org/10.1152/physrev.00045.2011

[6] Kohei K. (2010) Pathophysiology of Type 2 Diabetes and Its Treatment Policy. Japan Medical Association Journal, 53, 41-46.

[7] National Institute for Health and Clinical Excellence (2015) Type 2 Diabetes: The Management of Type 2 Diabetes. http://www.nice.org.uk/guidance/ng28/chapter/1-Recommendations\#drug-treatment-2

[8] American Diabetes Association (2003) Standards of Medical Care for Patients with Diabetes Mellitus. Diabetes Care, 26, S33-S50. http://dx.doi.org/10.2337/diacare.26.2007.S33

[9] Joseph, B. and Jini, D. (2013) Antidiabetic Effects of Momordica charantia (Bitter Melon) and Its Medicinal Potency. Asian Pacific Journal of Tropical Disease, 3, 93-102. http://dx.doi.org/10.1016/S2222-1808(13)60052-3

[10] Hosseini, S., Huseini, H.F., Larijani, B., Kazem, M., Alireza, N., Keramt, N., et al. (2014) The Hypoglycemic Effect of Juglans regia Leaves Aqueous Extract in Diabetic Patients: A First Human Trial. DARU Journal of Pharmaceutical Sciences, 22, 19. http://dx.doi.org/10.1186/2008-2231-22-19

[11] Fuangchan, A., Sonthisombat, P., Seubnukarn, T., Chanouan, R., Chotchaisuwat, P., Sirigulsatien, V., et al. (2011) Hypoglycemic Effect of Bitter Melon Compared with Metformin in Newly Diagnosed Type 2 Diabetes Patients. Journal of Ethnopharmacology, 134, 422-428. http://dx.doi.org/10.1016/j.jep.2010.12.045

[12] Grover, J.K. and Yadav, S.P. (2004) Pharmacological Actions and Potential Uses of Momordica charantia: A Review. Journal of Ethnopharmacology, 93, 123-132. http://dx.doi.org/10.1016/j.jep.2004.03.035

[13] Altinterim, B. (2012) Bitter Melon (Momordica charantia) and the Effects of Diabetes Disease. Journal of Agricultural Faculty of Uludag University, 26, 65-69.

[14] Nerurkar, P.V., Lee, Y.K., Motosue, M., Adeli, K. and Nerurkar, V.R. (2008) Momordica charantia (Bitter Melon) Reduces Plasma Apolipoprotein B-100 and Increases Hepatic Insulin Receptor Substrate and Phosphoinositide-3 Kinase Interactions. British Journal of Nutrition, 100, 751-759. http://dx.doi.org/10.1017/S0007114508937430 
[15] Jung, H.S. (2015) Clinical Implications of Glucose Variability: Chronic Complications of Diabetes. Endocrinology and Metabolism, 30, 167-174. http://dx.doi.org/10.3803/EnM.2015.30.2.167

[16] Pandey, A., Tripathi, P., Pandey, R., Srivatava, R. and Goswami, S. (2011) Alternative Therapies Useful in the Management of Diabetes: A Systematic Review. Journal of Pharmacy \& Bioallied Sciences, 3, 504-512. http://dx.doi.org/10.4103/0975-7406.90103

[17] Swinnen, S.G., Hoekstra, J.B. and De Vries, J.H. (2009) Insulin Therapy for Type 2 Diabetes. Diabetes Care, 32, S253-S259. http://dx.doi.org/10.2337/dc09-s318

[18] Pradeep, T. and Haranath, C. (2014) A Review on Diabetes Mellitus Type II. International Journal of Pharma Research \& Review, 3, 23-29.

[19] Okayasu, S., Kitaichi, K., Hori, A., Suwa, T., Horikawa, Y., Yamamoto, M., et al. (2012) The Evaluation of Risk Factors Associated with Adverse Drug Reactions by Metformin in Type 2 Diabetes Mellitus. Biological and Pharmaceutical Bulletin, 35, 933-937. http://dx.doi.org/10.1248/bpb.35.933

[20] Lorenzati, B., Zucco, C., Miglietta, S., Lamberti, F. and Bruno, G. (2010) Oral Hypoglycemic Drugs: Pathophysiological Basis of Their Mechanism of Action. Pharmaceuticals, 3, 3005-3020. http://dx.doi.org/10.3390/ph3093005

[21] Rahman, I., Bashir, M., Salman, M., Idrees, M. and Khan, M. (2011) Bitter Melon (Momordica charantia) Reduces Serum Sialic Acid in Type 2 Diabetics: Evidence to Delay the Process of Atherosclerosis. Chinese Medicine, 2, 125-129. http://dx.doi.org/10.4236/cm.2011.24021

[22] Rahman, I.U., Khan, R.U., Rahman, K.U. and Bashir, M. (2015) Lower Hypoglycemic but Higher Antiatherogenic Effects of Bitter Melon than Glibenclamide in Type 2 Diabetic Patients. Nutrition Journal, 14, 13. http://dx.doi.org/10.1186/1475-2891-14-13

[23] Haddadinezhad, S. and Ghazaleh, N. (2010) Relation of Fasting and Postprandial and Plasma Glucose with HemoglobinA1c in Diabetics. International Journal of Diabetes in Developing Countries, 30, 8-10. http://dx.doi.org/10.4103/0973-3930.60002

[24] Dans, A.M., Villarruz, M.V., Jimeno, C.A., Javelosa, M.A., Chua, J., Bautista, R., et al. (2007) The Effect of Momordica charantia Capsule Preparation on Glycemic Control in Type 2 Diabetes Mellitus Needs Further Studies. Journal of Clinical Epidemiology, 60, 554-559. http://dx.doi.org/10.1016/j.jclinepi.2006.07.009

[25] Zanker, K.S., Mang, B., Wolters, M. and Hahn, A. (2012) Personalized Diabetes and Cancer Medicine: A Rationale for Anti-Diabetic Nutrition (Bitter Melon) in a Supportive Setting. Current Cancer Therapy Reviews, 8, 66-77. http://dx.doi.org/10.2174/157339412799462521

[26] Sutherland, E.W., Robison, G.A. and Butcher, R.W. (1968) Some Aspects of the Biological Role of Adenosine 3’,5’-Monophosphate (Cyclic AMP). Circulation, 37, 279-306. http://dx.doi.org/10.1161/01.CIR.37.2.279

[27] Berg, J.M., Tymoczko, J.L. and Stryer, L. (2002) Biochemistry. 5th Edition. Section 16.1, Glycolysis Is an Energy-Conversion Pathway in Many Organisms. WH Freeman, New York. http://www.ncbi.nlm.nih.gov/books/NBK22593/

[28] Rhoades, R.A. (2013) Medical Physiology: Principles for Clinical Medicine. 4th Edition. Chapter 27, Liver Physiology, Lippincott Williams \& Wilkins, Baltimore, 536.

[29] Zea, C.J., Camci-Unal, G. and Pohl, N.L. (2008) Thermodynamics of Binding of Divalent Magnesium and Manganese to Uridine Phosphates: Implications for Diabetes-Related Hypomagnesaemia and Carbohydrate Biocatalysis. Chemistry Central Journal, 2, 15. http://dx.doi.org/10.1186/1752-153X-2-15

[30] King, M.W. (2015) Glycoproteins. http://themedicalbiochemistrypage.org/glycoproteins.php\#oglcnac 